\title{
Statin-induced Myopathy
}

\author{
Anthony J. Dalby \\ Milpark Hospital, Johannesburg, South Africa \\ Address for correspondence: \\ Anthony J. Dalby \\ Suite $C$ \\ Milpark Hospital \\ Guild Road \\ Parktown West \\ Johannesburg \\ 2193 \\ South Africa \\ Email: \\ ajd@hot.co.za
}

\section{INTRODUCTION}

Dyslipidaemia is a major risk factor for the development of atherosclerotic cardiovascular disease, the most frequent cause of death worldwide. A large number of trials have demonstrated that reducing LDL cholesterol with 3-hydroxy-3methyl-glutaryl-CoA (HMGCoA) reductase inhibitors (commonly known as statins) is both safe and effective in decreasing the risk of myocardial infarction, stroke and death. These findings have been collated by the Cholesterol Trialists' Collaboration $^{(1)}$ and both local(2) and international( ${ }^{(3,4)}$ guidelines on cardiovascular prevention have incorporated recommendations supporting the use of statin therapy in primary and secondary prevention. Broadly, these guidelines support the concept of "lower is better" and recommend high dose potent statin treatment ${ }^{(4)}$ for patients with established disease, patient with diabetes, and those with a very high risk of developing cardiovascular disease, aiming to achieve levels of LDL cholesterol $<1.8 \mathrm{mmol} / \mathrm{L}^{(2,3)}$ Moderate doses of statin are recommended for those at less than very high risk. A significant uptake of this advice by the medical community has resulted in the widespread prescription of statins.

\section{STATIN ASSOCIATED MYOPATHY}

The occurrence of myalgia without CK elevation during statin therapy was not widely appreciated before 2002. ${ }^{(5)}$ However, two thirds of the side-effects of statin treatment arise from muscle symptoms. ${ }^{(6)}$ The term statin-associated myopathy is used here to encompass all muscle problems that arise during

\section{ABSTRACT}

\begin{abstract}
Atherosclerotic cardiovascular disease (ASCVD) is the leading cause of death worldwide. HMGCoA reductase inhibitors or "statins" reduce low density lipoprotein cholesterol and reduce the risks of myocardial infarction, stroke and death in the presence of dyslipidaemia. In consequence, statins are prescribed to a large number of patients requiring primary or secondary prevention. A variety of side-effects may arise during the course of statin treatment which interfere with the quality of patients' lives and reduce their compliance with therapy. Muscle symptoms constitute the most common of these side-effects and are the most frequent reason for discontinuing treatment. This review defines the muscle, tendon and joint disorders encountered by patients on statin treatment, their possible relationship to statin use, and the factors that facilitate the emergence of symptoms. The subtypes of statin myopathy are discussed and a general definition of statin myopathy is offered. Expert advice on managing statin myopathy is summarised. SAHeart 2015;12:14-18
\end{abstract}

statin use. In order of decreasing frequency and increasing severity they are:

Asymptomatic elevation in serum creatine kinase (CK) level,

myalgia (without elevation in the CK),

myositis (with an elevated CK) and

rhabdomyolysis. ${ }^{(6)}$

Rhabdomyolysis is defined as muscle symptoms associated with a marked elevation in CK (typically much in excess of IOX normal) and an elevation in serum creatinine and myoglobinuria. It is rarely encountered with statin monotherapy. ${ }^{(7)}$ An excess of rhabdomyolysis of $0.01 \%$ vs. placebo was reported in trials. ${ }^{(8)}$ Death from rhabdomyolysis with statin therapy has been encountered in $<1$ in a million prescriptions. ${ }^{(7)}$ Notwithstanding, unless recognised and treated early, rhabdomyolysis carries a dire prognosis and must be excluded promptly when patients on statins present with muscle pain.

In addition to the above, a very rare condition, necrotising immune-mediated statin myopathy (IMSM), has been recognised recently. ${ }^{(9)}$ This appears to be a genetically mediated, autoimmune reaction caused or unmasked by statin therapy. IMSM 
is characterised by persistent or worsening symptoms despite the withdrawal of treatment, elevation in CK, and dependence upon immunosuppressive therapy to prevent relapse.

The mechanism/s by which statins induce these changes in skeletal muscle are not fully understood. Drug interactions, genetic predisposition, depletion of metabolites in the mevalonate pathway, mitochondrial dysfunction, dysregulation of apoptosis and protein degradation and immune mechanisms have all been implicated. ${ }^{(10)}$

The muscle ache and/or muscle weakness associated with a statin myopathy is most often bilateral, but may be asymmetrical. It predominantly affects large muscle groups around the shoulders, hips, thighs and/or calves. Although the patient cannot usually identify a precipitating factor, recent strenuous or unaccustomed exercise, or an alteration in medication may be reported. ${ }^{(10)}$ There are no specific clinical signs that accompany the pain. The serum CK should be measured and may be elevated.

While the patient's symptoms may be tolerable and not necessarily severe, statin myopathy reduces adherence to treatment, limits physical activity, reduces the quality of life, and may lead to discontinuation of statin therapy, ultimately depriving the at-risk dyslipidaemic patient of the clinical benefits of statin treatment. ${ }^{(8)}$

The incidence of muscle aches (myalgia), muscle cramps ${ }^{(1)}$ and weakness is generally reported to be in the region of $7-11 \%$ of patients taking statin monotherapy ${ }^{(8)}$ but is encountered more frequently when drugs that interact with statins are given concomitantly, or in the presence of certain co-morbid conditions. However, there is no consensus on the frequency at which statin myopathy occurs. The observed incidence of muscle symptoms is greater in clinical practice than was noted in clinical trials. This discrepancy may arise from the non-specific nature of the symptoms, the lack of a clear definition of statin myopathy, or from the design of some clinical trials which excluded patients who developed muscle symptoms during a run-in period or did not specifically require the solicitation of muscle problems. ${ }^{(12)}$ Because many patients requiring statin treatment are middle aged, it may be difficult to establish whether their symptoms are caused by the statin therapy or originate from unrelated musculoskeletal conditions. Although placebo controlled clinical trials have recorded a higher rate of myalgia in patients treated with statins, the placebo groups in these trials had nearly as many muscle symptoms as the actively treated groups. An extensive review of published trials concluded that both patients and physicians overestimate the frequency of myalgia associated with statins and that muscle symptoms are much less common than the $25 \%$ incidence stated in certain reports. ${ }^{(12)}$
Myalgia is the most common reason that patients discontinue statin treatment. On average, symptoms appear within 4 weeks of starting treatment ${ }^{(13,14)}$ but their onset may be delayed for up to 2 years. ${ }^{(15)}$ The frequency of statin myopathy does not increase with longer term treatment. ${ }^{(16)}$ The reported incidence of muscle symptoms in statin trials suggests that the frequency of symptoms relates to the potency of the statin employed. (12) There may be a relationship between the incidence of statinassociated myopathy and the extent of reduction in total and LDL cholestero('17) although this is not supported by the results of clinical trials or clinical experience. ${ }^{(18)}$

There is no standard definition of statin myopathy nor is there any test that conclusively proves the diagnosis. The STOMP trial defined statin-associated myalgia as new or increased muscle pain, cramps, or aching unassociated with exercise that persisted for 2 weeks and resolved within 2 weeks of stopping the drug and reoccurred within 4 weeks of restarting the medication.(14) STOMP tested atorvastatin $80 \mathrm{mg}$ daily vs. placebo for 6 months in healthy subjects, demonstrating an increase in muscle symptoms ( $9.4 \%$ vs. $4.8 \%$ ) and a modest rise in CK on atorvastatin therapy (about $20 \mathrm{U} / \mathrm{L}$ ). The rise in CK suggested a low level of skeletal muscle injury that was not related to the emergence of symptoms. STOMP found that muscle strength, endurance or aerobic performance were impaired only if the patient fulfilled the criteria for statininduced myalgia.

Statin myopathy arises from complex interactions between the specific statin, concurrent disease, genetic predisposition and concomitant treatment. ${ }^{(19)}$ Although it is a class effect, the incidence of myopathy varies amongst the various members of the drug class. Myopathy is seen 4 times more frequently with simvastatin $80 \mathrm{mg}$ compared to atorvastatin $80 \mathrm{mg} .{ }^{(20)}$ Whereas simvastatin, atorvastatin and rosuvastatin are more commonly associated with statin myopathy, it is rarely found in association with the less potent agents viz. fluvastatin or pravastatin ${ }^{(21)}$ or pitivastatin.(22) Statin myopathy may be aggravated by strenuous exercise and may affect up to $75 \%$ of athletes taking statins. ${ }^{(23)}$ Vulnerability to statin myopathy is increased in persons of Asian extraction, and also by female sex, advanced age, small body size and frailty. Chronic comorbidities such as diabetes, chronic kidney injury, hepatic dysfunction, untreated hypothyroidism and heavy alcohol intake are associated with a higher risk of myopathy. Acute perturbations such as drug abuse, acute infection, extensive surgery and major trauma may also be implicated. ${ }^{(19)}$ Interactions between statins and other drugs, notably the inhibitors of cytochrome P450 CYP3A4 may result in the development of myopathy. This group of drugs includes, but is not limited to, the protease inhibitors, ciclosporin, amiodarone, the non-dihidropyridine calcium channel blockers (verapamil and diltiazem), digoxin, warfarin, the macrolide 
antibiotics, the azole antifungal agents, the antidepressant nefazodone, the histamine $\mathrm{H} 2$ receptor blockers, omeprazole and grapefruit juice consumption in excess of I litre a day. ${ }^{(18)}$ Because fluvastatin is metabolised by CYP2C9 and not by CYP3A4, it is free of this interaction. This difference in metabolism and the lower potency of fluvastatin are thought to account for the lower incidence of muscle symptoms encountered with its use. ${ }^{(13)}$ The addition of a fibrate (especially gemfibrozil) to statin therapy increases the likelihood of myopathy by its action on a variety of mechanisms. ${ }^{(19)}$ In addition, certain genetic polymorphisms (viz. SLCOIBI and $A B C G 2$ ) increase the predisposition to statin myopathy ${ }^{(24,25)}$ by reducing the hepatic uptake of statin and exposing skeletal muscle to greater concentrations of the drug.

Despite the frequent occurrence of statin-induced myopathy, the potential to reduce the risk of a clinical cardiovascular event (number needed to treat=27) is far greater than the potential for raising the $\mathrm{CK}>\mathrm{IOX}$ normal (number needed to harm $[N N H]=3.400)$ or for rhabdomyolysis $(\mathrm{NNH}=7.428)$. ${ }^{(26)}$ The significant difference in favour of continuing treatment emphasises the importance of exploring alternative strategies for controlling the cholesterol of patients who are intolerant of standard statin therapy.

\section{MANAGEMENT}

No adequately powered trial has been carried out in patients with statin myopathy that has demonstrated a reliable method for managing the condition. Thus, in the absence of firm evidence, the following recommendations concerning the management of statin myopathy are based on the published opinions of experts in the field.

Review of the original indication for treatment: The emergence of muscle symptoms should occasion a reevaluation of the initial indication for treatment. Particularly in primary prevention in moderate risk patients, the need for statin therapy should be carefully reconsidered and the patient's risk score reassessed. It is prudent not to administer statins to patients who are unlikely to derive significant benefit in the longer term. In such instances, statin treatment should be substituted with a lifestyle programme that incorporates exercise and a strict dietary regimen. On the other hand, because "lower is better," in high risk patients requiring secondary prevention, it may be more appropriate to consider altering statin to a less aggressive regimen, even though the lipid target may not be fully met.

- Measure CK: In patients with CK levels 3-5X normal, it is safe to continue statin treatment provided that regular CK monitoring is undertaken thereafter. It is appropriate to discontinue the patient's statin treatment when CK levels exceed $5 X$ normal if there is no alternative explanation for the elevation e.g. recent strenuous or unaccustomed exercise.

- Review of concomitant medication: The patient's list of medications should be carefully reviewed to discover any potential for statin-drug interactions (Table I). Whenever possible, the patient's prescription should be adjusted to avoid these interactions.

- Assess the patient's exercise programme: Myopathic symptoms may be ameliorated by advising a reduction in exercise intensity in patients who exercise actively.

Consider a drug holiday: In patients with intolerable symptoms, temporary discontinuation of the statin for 2-3 weeks should be proposed with a re-challenge following this break. It should then be possible to establish whether symptoms were improved or unaffected by withdrawing the statin, and whether symptoms return on re-challenge.

TABLE I: Factors increasing the risk of statin myopathy.

\begin{tabular}{|l|l|}
\hline \hline Choice of statin & $\begin{array}{l}\text { Simvastatin in a dose }>40 \mathrm{mg} \text { daily } \\
\text { More potent statins vs. less potent statins }\end{array}$ \\
\hline Diet & $\begin{array}{l}\text { Grapefruit juice consumption in excess of } \\
\text { I litre a day }\end{array}$ \\
\hline Strenuous exercise & \\
\hline Demographic factors & Asian extraction \\
& Female sex \\
& $\begin{array}{l}\text { Advanced age } \\
\text { Small body size }\end{array}$ \\
& Frailty \\
\hline Chronic comorbidity & $\begin{array}{l}\text { Diabetes } \\
\text { Chronic kidney injury } \\
\text { Hepatic dysfunction } \\
\text { Untreated hypothyroidism } \\
\text { Heavy alcohol intake }\end{array}$ \\
& $\begin{array}{l}\text { Acute infection } \\
\text { Extensive surgery } \\
\text { Major trauma }\end{array}$ \\
Drug abuse \\
\hline Acute illness & $\begin{array}{l}\text { Protease inhibitors } \\
\text { Ciclosporin } \\
\text { Amiodarone } \\
\text { Non-DHP calcium channel blockers } \\
\text { (verapamil and diltiazem) } \\
\text { Digoxin } \\
\text { Warfarin } \\
\text { Macrolide antibiotics } \\
\text { Azole antifungal agents } \\
\text { Histamine H2 receptor blockers } \\
\text { Omeprazole } \\
\text { Fibates, especially gemfibrozil }\end{array}$ \\
\hline SLCOIBI \\
ABCG2 \\
\hline Concomitant medication polymorphism
\end{tabular}

$\mathrm{DHP}=$ dihydropyridine 
A Canadian group undertook a "trial of I" to establish whether the muscle symptoms were truly related to statin use. Their method exposed the statin-intolerant patient to courses of the various statins, alternating with periods of placebo treatment, while continuously assessing the intensity of symptoms. In their experience, the majority of myopathic symptoms could not be related to statin treatment. ${ }^{(27)}$ Statin treatment may be recommenced in patients whose symptoms do not improve after a period of withdrawal, dependent upon a risk-benefit evaluation. ${ }^{(10)}$

Consider altering the statin treatment: Existing statin treatment may be altered by:

- reducing the dose,

- changing the frequency of dosing to alternate days or to twice weekly when using a long-acting potent statin (high dose atorvastatin or rosuvastatin) or

- switching to a less potent statin (fluvastatin, pravastatin or pitivastatin).

- Combination treatment: A low dose of statin in combination with ezetimibe may reduce the intensity of myalgia while effecting a significant reduction in LDL cholesterol. Recently the IMPROVE-IT outcome study demonstrated small, but significant, cardiovascular benefit when ezetimibe was added to simvastatin treatment (American Heart Association Annual Meeting, Chicago, 2014).

Non-statin monotherapy: Ezetimibe monotherapy may be tried if the patient remains intolerant of any statin treatment. A 10-15\% fall in LDL cholesterol can be anticipated, significantly less than that achieved by statins. The bile acid sequestrants, such as cholestyramine and colesevelam, are not available in South Africa. Niacin or fibrate monotherapy is not recommended as these agents have little or no effect on LDL cholesterol and have not been shown to improve cardiovascular outcomes.

Over-the-counter preparations: Red rice yeast extract and plant sterols effect small reductions in LDL cholesterol and may be tried as an alternative in the patient who is totally statin intolerant. ${ }^{(20)}$

Supplementation of Vitamin D in deficient patients: Nonrandomised studies have reported that muscle symptoms may resolve in Vitamin D deficient patients presenting with statin-induced myopathy after supplementation with Vitamin D $50000 \cup$ weekly. ${ }^{(10)}$

Supplementation with coenzyme Q10: Although it has not been shown conclusively that CoQ10 improves statin myopathy, certain patients report an improvement in their muscle symptoms after adding CoQ $10200 \mathrm{mg}$ daily to their statin treatment. Whether the improvement represents individual responsiveness or placebo effect is not known.
However, as CoQIO has no known harmful effects, a trial of supplementation could be considered in patients refractory to other measures. ${ }^{(28)}$

- Lipid-lowering agents undergoing development: The apolipoprotein BI00 synthesis inhibitor mipomersein and the proprotein convertase subtilisin/kexin type 9 (PCSK9) inhibitors such as evolucumab and alirocumab are both better tolerated than statins in statin-intolerant patients. However, as mipomersein use was associated with elevations in hepatic transaminase levels and steatosis, PCSK9 inhibition presently seems to be the more likely future substitute for statin treatment. ${ }^{(29,30)}$

\section{CONCLUSION}

Though myositis and rhabdomyolysis are extremely rare events during statin therapy, many patients will complain of myalgia when taking statin treatment. Although substantial experience suggests that much of the muscle ache encountered may not relate to statin treatment, the condition requires management nonetheless to ensure that patients with established atherosclerotic vascular disease, and those at high risk thereof, continue to receive treatment to reduce their LDL cholesterol. In many instances, it will be possible to continue statin therapy.

\author{
Disclosure: This review was initially undertaken at \\ the request of DiscoveryHealth, which supported \\ the author with a writing grant.
}

The author gratefully acknowledges Mr Q. Barber of AstraZeneca for his assistance in accessing a number of the references quoted herein. 


\section{REFERENCES}

I. Cholesterol trialists' collaboration. Efficacy and safety of more intensive lowering of LDL cholesterol: A meta-analysis of data from 170000 participants in 26 randomised trials. Lancet 20 10;376(9753): 1670-1681.

2. Klug E, for South African Heart Association (SA Heart) \& Lipid and Atherosclerosis Society of Southern Africa (LASSA). South African dyslipidaemia guideline consensus statement. S Afr Med J 2012;102:178-87.

3. The task force for the management of dyslipidaemias of the European Society of Cardiology (ESC) and the European Atherosclerosis Society (EAS). ESC/EAS guidelines for the management of dyslipidaemias. Eur Heart J 2011; 32:1769-1818.

4. Stone NJ, Robinson J, Lichtenstein AH, et al. 2013 ACC/AHA guideline on the treatment of blood cholesterol to reduce atherosclerotic cardiovascular risk in adults: A report of the American College of Cardiology/ American Heart Association task force on practice guidelines. Circulation 20|4; | 29:SI-45.

5. Phillips PS, Haas RH, Bannykh S, et al. Statin-associated myopathy with normal creatine kinase levels. Ann Intern Med 2002; I 3:58 I-5.

6. Raju SB, Varghese K, Medhu K. Management of statin intolerance. Ind J Endocrinol Metab 2013;17:977-82.

7. Pasternak RC, Smith SC, Bairey-Merz CN, et al. ACC/AHA/NHLBI Clinical advisory on the use and safety of statins. Circulation 2002;106:1024-8.

8. Chapman MJ, Carrie A. Mechanism of statin-induced myopathy. A role for the ubiquitin-proteosome pathway? Arterioscl Thromb and Vasc Biol 2005; 25:244I-4

9. Needham M, Fabian V, Knezevic W, et al. Progressive myopathy with upregulation of MHC-I associated with statin therapy. Neuromusc Disord 2007; 17:194-200

10. Auer J, Sinzinger H, Franklin B, et al. Muscle- and skeletal-related side-effects of statins: tip of the iceberg? Eur J Prevent Cardiol Sept 17, 2014; publ. online.

II. Thompson PD, Parker B. Statins, exercise and exercise training. J Am Coll Cardiol 2013;62:7|5-6.

12. Ganga HV, Slim HB, Thompson PD. A systematic review of statin-induced muscle problems in clinical trials. Am Heart J 2014;168:6-15.

13. Bruckert E, Hayem G, Dejager S, et al. Statin therapy in hyperlipidemic patients: The PRIMO study. Cardiovasc Drugs and Ther 2005; 19:403-4I4

14. Parker BA, Capizzi JA, Grimaldi AS, et al. Effect of statins on skeletal muscle function. Circulation 2013;127:96-103

15. Bellosta S, Paoletti R, Corsini A. Safety of statins. Focus on clinical pharmacokinetics and drug interactions. Circulation 2004; 109 [suppl III]:III-50-III-57.

16. Mehrotra S, Kim K, Liebivitz D. Myopathy prevalence in statin treated patients with comorbidities and combination therapies. Am Coll Cardiol Scientific Sessions 2012; Abstr. 2536-634.

17. Morales DCV, Parker B, Lorson L, et al. Greater reductions in total and low density lipoprotein cholesterol are associated with concomitant development of statin myopathy. Am Coll Cardiol Scientific Sessions 201 I; Abstr. II13-278.

18. Thompson PD, Clarkson PM, Rosenson RS. An assessment of statin safety by muscle experts. Am J Cardiol 2006;97[suppl]:69C-76C.

19. Chatzizisis YS, Koskinas KC, Misirli G, et al. Risk factors and drug interactions predisposing to statin-induced myopathy. Implications for risk assessment, prevention and treatment. Drug Saf 2010;33:171-187.

20. Davidson MH, Robinson JG. Safety of aggressive lipid management. J Am Coll Cardiol 2007;49:1753-62
21. Eckel RH. Approach to the patient who is intolerant of statin therapy. J Clin Endocrinol Metab 2010;95:2015-22.

22. Holloway B, Jensen C, Steinmann $\mathrm{H}$, et al. Tolerability and efficacy of pitivastatin among hyperlipidemic patients intolerant to at least 2 other statins. Am Coll Cardiol Scientific Sessions 2013; Abstr. 1275-16.

23. Larsen S, Stride N, Hey-Mogensen M, et al. Simvastatin effects on skeletal muscle. relation to decreased mitochondrial function and glucose intolerance. | Am Coll Cardiol 2013;61:44-53.

24. Voora D, Shah SH, Spasojevic I, et al. The SLCOIBI*5 genetic variant is associated with statin-induced side effects. J Am Coll Cardiol 2009; 54:1609-16.

25. Generaux GT, Bonomo FM, Johnson M, et al. Impact of SLCOIBI (OATPIBI) and ABCG2 (BCRP) genetic polymorphisms and inhibition on LDL-C lowering and myopathy of statins. Xenobiotica 201 1;41:639-51.

26. Kohli P, Cannon CP. A new approach to managing the "statin-intolerant" patient? Eur Heart J 20 I2;33: 1040-1043.

27. Joy TR, Monjed A, Zou GY, et al. N-of-I (single-patient) trials for statinrelated myalgia. Ann Intern Med 2014;160:301-310.

28. Marcoff L, Thompson PD. The role of coenzyme Q10 in statin-associated myopathy. A systematic review. J Am Coll Cardiol 2007;49:2231-7.

29. Visser ME, Wagener G, Baker BF, et al. Mipomersen, an apolipoprotein B synthesis inhibitor, lowers low-density lipoprotein cholesterol in high-risk statin-intolerant patients: A randomised, double-blind, placebo-controlled trial. Eur Heart | 20 12;33: I | 42-1 | 49

30. Stroes E, Colquhoun D, Sullivan D, et al. Anti-PCSK9 antibody effectively lowers cholesterol in patients with statin intolerance. The GAUSS-2 randomised, placebo-controlled phase 3 clinical trial of evolocumab. J Am Coll Cardiol 2014:63:254|-8. 